\title{
Harmonic minimization waveforms for modulated heating experiments at HAARP
}

\author{
G. Jin, ${ }^{1}$ M. Spasojevic, ${ }^{1}$ M. B. Cohen, ${ }^{1}$ and U. S. Inan ${ }^{1,2}$ \\ Received 6 July 2012; revised 8 October 2012; accepted 9 October 2012; published 22 November 2012.
}

[1] Modulated High Frequency (few MHz) heating of the $D$-region ionosphere under the auroral electrojet is capable of generating extremely low frequency (ELF) radio waves in the few kilohertz range by affecting the conductivity of the $D$-region. The HF heating is nonlinear and results in the generation of harmonics at integer multiples of the ELF modulation frequency with $\sim 1 \%$ of the total power outside the fundamental when sinusoidal amplitude modulation is applied to the HF carrier. For the purpose of harmonic minimization, we present a modulation scheme designed to create a sinusoidal change in the Hall conductivity at a particular altitude in the ionosphere. The modulation waveform is generated by inverting a numerical HF heating model, starting from the desired conductivity time series, and obtaining the HF power envelope at the bottom of the ionosphere. The inverted envelopes (referred to as inv-sin waveforms) are highly sensitive to the assumed ionospheric density profile and simulations indicate that these waveforms have less harmonic distortion compared to sinusoidal modulation when the actual ionospheric density is similar to or less dense than the one assumed. Experimental results indicate that sinusoidal amplitude modulation may still be preferred since it is more robust to the highly variable ionospheric profile while square wave modulation is more efficient in generation of ELF waves when harmonic distortion is not important. The inv-sin waveforms are more efficient than sinusoidal modulation while still suffering from less harmonic distortion than square wave modulation suggesting a tradeoff between harmonic distortion and ELF generation efficiency.

Citation: Jin, G., M. Spasojevic, M. B. Cohen, and U. S. Inan (2012), Harmonic minimization waveforms for modulated heating experiments at HAARP, J. Geophys. Res., 117, A11315, doi:10.1029/2012JA018102.

\section{Introduction}

[2] Modulated heating of the $D$-region ionosphere can generate waves in the extremely low frequency (ELF, $300 \mathrm{~Hz}$ to $3 \mathrm{kHz}$ ) and very low frequency (VLF, 3-30 kHz) range. Amplitude modulation of a high-frequency (HF, 3-30 MHz) beam modulates the temperature and conductivity of the lower ionosphere. When performed under natural horizontal ionospheric currents, this heating leads to a time-varying current that radiates at the modulation frequency. Examples of modulated heating experiments include Getmantsev et al. [1974], Kapustin et al. [1977], Rietveld et al. [1987], Barr and Stubbe [1984], and Milikh et al. [1999]. The High Frequency Active Auroral Research Program (HAARP) facility

\footnotetext{
${ }^{1}$ Department of Electrical Engineering, Stanford University, Stanford, California, USA.

${ }^{2}$ Department of Electrical Engineering, Koç University, Istanbul, Turkey.

Corresponding author: G. Jin, Department of Electrical Engineering, Stanford University, 350 Serra Mall, Rm. 356, Stanford, CA 94305, USA. (gj36@stanford.edu)

(C)2012. American Geophysical Union. All Rights Reserved. 0148-0227/12/2012JA018102
}

in Gakona, Alaska is currently the most powerful ionospheric heater with 3.6 MW of HF power and over $1 \mathrm{GW}$ ERP. Its capabilities have led to many experiments which have advanced our understanding of wave injection into the magnetosphere [Golkowski et al., 2008], the plasma processes involved in modulated heating [Moore et al., 2006], and more novel techniques for generating ELF waves [Cohen et al., 2010b; Papadopoulos et al., 2011].

[3] By utilizing natural currents, modulated heating eases the challenges of directly constructing the large antennas needed to radiate efficiently at such long wavelengths. However, there are other difficulties with using modulated ionospheric heating as a source of ELF/VLF waves. One example is the nonlinear conversion between the HF power and the conductivity changes in the ionosphere. This nonlinear distortion leads to radiation of harmonics at integer multiples of the fundamental modulation frequency [Stubbe et al., 1982]. These harmonics consume bandwidth and can interfere with other signals in communications applications. Naval communications are a particularly important application of ELF/VLF waves as these waves can be used to transmit messages to submerged submarines. Although modulated heating is very inefficient in generating ELF waves, so are more traditional horizontal wire antennas [e.g., Bernstein et al., 1974], and these two techniques 
are currently the only option for generating waves below a few $\mathrm{kHz}$. Thus, techniques for minimizing or at least characterizing the generated harmonics can be helpful in designing potential communication systems.

[4] The harmonics generated in modulated heating are influenced by several variables. One of the most important is the waveform used to modulate the HF power as the harmonic content of the waveform is a component of the harmonic content of the radiated ELF waves. Square wave modulation, where the HF beam is turned on and off at an ELF frequency is a simple modulation waveform that intrinsically has strong odd harmonics but no even harmonics. James [1985] presented observations and simulations of harmonic content for the heater at Tromsø, Norway using an HF frequency of $2.8 \mathrm{MHz}$ with $\mathrm{X}$ - and $\mathrm{O}$ - mode polarizations and square-wave amplitude modulation at $525 \mathrm{~Hz}$. In modeling with daytime electron density and temperature profiles, they noted a change in the conductivity spectrum due to the fact that heating and cooling time constants increased with altitude. Below $65 \mathrm{~km}$, the conductivity closely approximated a square wave with very weak even harmonics ( $>30 \mathrm{~dB}$ below the fundamental). Between $65-85 \mathrm{~km}$, the heating and cooling time constants are comparable to the ELF period ( $\sim 1 \mathrm{~ms})$, hence the evolution of the conductivity with time became a double-exponential with stronger even harmonics ( $\sim 15 \mathrm{~dB}$ below the fundamental). Above $85 \mathrm{~km}$, the recovery time is substantially longer than the ELF period so that the conductivity function is a triangular wave with weaker even harmonics again. The third harmonic decreased from $10 \mathrm{~dB}$ below the fundamental at $60 \mathrm{~km}$ to nearly $20 \mathrm{~dB}$ below at $100 \mathrm{~km}$. Nighttime results were similar except the transition to a more triangular wave occurs at $95 \mathrm{~km}$. These simulations failed to reproduce experimental results shown in James et al. [1984] where ground receivers $17 \mathrm{~km}$ from the heater detected a third harmonic nearly the same amplitude as the fundamental and a fifth harmonic only $4 \mathrm{~dB}$ below the fundamental. Even harmonics were approximately $10 \mathrm{~dB}$ below the fundamental. Measurements on the ISIS satellite corresponded more closely to the simulations and the authors suggested that waveguide effects were partly responsible for the discrepancies in ground observations. Papadopoulos et al. [2003] noted similar results at HAARP where the harmonic content of received signals on the ground were stronger than would be expected from a square wave when a $3.3 \mathrm{MHz} 960 \mathrm{~kW}$ HF beam was used. Barr and Stubbe [1993] attempts to correct for waveguide effects by normalizing the received amplitude of each harmonic with the received amplitude of a wave with a fundamental frequency that was the same as the harmonic. After normalizing, the harmonic content was similar to that of a square wave though there are still even harmonics $29 \mathrm{~dB}$ below the fundamental.

[5] A second important factor influencing the spectrum of the radiated signal is the nonlinearity of the HF heating process, such that scaling the HF power does not scale the generated signal. For this reason, sinusoidal modulation, though it does not have any inherent harmonic content, still results in substantial received harmonics. For example, Oikarinen et al. [1997] showed significant harmonic content of a $1375 \mathrm{~Hz}$ signal generated with sinusoidal modulation. Moore et al. [2006] conducted experiments at HAARP using a sinusoidally modulated $3.25 \mathrm{MHz}$ beam and examined the amplitudes of the fundamental, second, and third harmonics as a function of ELF frequency as well as HF power (up to $771 \mathrm{~kW}$ ). The second harmonic was typically $\sim 8 \mathrm{~dB}$ below the fundamental with the third harmonic $>15 \mathrm{~dB}$ below depending on the HF power. Numerical models also agreed with those experimental results.

[6] In addition to minimizing harmonic distortion, maximizing absolute received power is also an important consideration in choosing a modulation waveform. For example, square modulation waveforms can also have variable duty cycles, that is, the percentage of an ELF period that the HF beam is turned on, with different duty cycles resulting in more or less received power. Cohen [2009] showed the harmonic content when an HF beam is modulated with a square wave at various duty cycles. A $40 \%$ duty cycle resulted in the highest received fundamental amplitude because the time constant for electron heating is faster than that for cooling. The HF beam needs to be on only long enough for the electron temperature to reach saturation. Leaving the beam off for a slightly longer time allows the electrons more time to cool to their ambient temperature. The smallest duty cycle used $(15 \%)$ was the most efficient in the fundamental, where efficiency is defined as the fundamental amplitude divided by the duty cycle.

[7] Thus far there has not been a published work that directly compares the harmonic content and received power of standard modulation waveforms or an attempt to use numerical modeling to create novel waveforms that minimize generated harmonics. In this paper, we describe a technique for using a numerical HF heating model to determine the waveform required to induce a sinusoidal variation in the Hall conductivity at a particular altitude, essentially via predistortion. We introduce the HF heating model and show how it can be adapted to compute these waveforms. Next we conduct simulations to verify the waveforms are effective in reducing harmonic distortion and examine how sensitive the distortion performance is to the assumption of the ionospheric electron density profile. For the first time, we present experimental results and compare the measured harmonic content of ELF waves generated using these waveforms to ones generated using traditional sinusoidal modulation and square wave modulation of varying duty cycles. In addition to harmonic content, we also compare the received power and efficiency of each modulation waveform. This comparison shows a tradeoff between square wave modulation, with high efficiency but high distortion, sine wave modulation, with low efficiency and low distortion, and inv-sin modulation with intermediate levels of both.

\section{Methodology}

[8] An HF heating model is used to compute the change in the ionospheric conductivity over a range of altitudes when the ionosphere is heated with a given HF power envelope. The model we use is described in detail in Cohen et al. [2010a] and builds on the work of Tomko et al. [1980], Rodriguez [1994], Moore [2007], and Payne [2007]. We outline the procedure used by the model to compute conductivity from the HF heating power before examining how this procedure can be 
reversed to compute the HF heating power required to obtain a sinusoidal conductivity.

[9] The relationship between HF power, $S$, and electron temperature, $T_{e}$, is given by the energy balance equation

$$
\frac{3}{2} N_{e} k_{B} \frac{d T_{e}}{d t}=2 k \chi S-L_{e}
$$

$N_{e}$ is the electron density, $k_{B}$ is Boltzmann's constant, $k$ is the HF wave number, $-\chi$ is the imaginary component of the refractive index, and $L_{e}$ represents the electron energy loss rate. The loss rate is a sum of the losses from elastic collisions with molecular nitrogen and oxygen [Banks, 1966], as well as rotational excitation [Mentzoni and Row, 1963; Dalgarno et al., 1968] and vibrational excitation [Stubbe and Varnum, 1972], compiled in Rodriguez [1994]. The molecular nitrogen and oxygen concentration, as well as the ambient temperature are taken from the MSISE-90 model [Hedin, 1991]. This differential equation can be solved numerically to give the electron temperature as a function of time.

[10] The modified collision frequency is then given by the sum of

$$
\begin{gathered}
\nu_{e f f}^{\mathrm{N}_{2}}=\frac{5}{3} \cdot 2.33 \times 10^{-17} N_{\mathrm{N}_{2}}\left(1-1.25 \times 10^{-4} T_{e}\right) T_{e} \\
\nu_{e f f}^{\mathrm{O}_{2}}=\frac{5}{3} \cdot 1.82 \times 10^{-16} N_{\mathrm{O}_{2}}\left(1+1.36 \times 10^{-2} \sqrt{T_{e}}\right) \sqrt{T_{e}}
\end{gathered}
$$

from Banks [1966] with the 5/3 term derived from Sen and Wyller [1960]. $N_{\mathrm{N}_{2}}$ and $N_{\mathrm{O}_{2}}$ are the concentrations of molecular nitrogen and oxygen. Finally, the Hall and Pedersen conductivities can be written as

$$
\begin{gathered}
\sigma_{\mathrm{P}}=\frac{4 \pi q^{2}}{3 m_{e}} \int \frac{\nu_{a v} v_{e}^{3}}{\nu_{a v}^{2}+\omega_{c e}^{2}} \frac{\partial f_{e}}{\partial v_{e}} \mathrm{~d} v_{e} \\
\sigma_{\mathrm{H}}=\frac{4 \pi q^{2}}{3 m_{e}} \int \frac{\omega_{c e} v_{e}^{3}}{\nu_{a v}^{2}+\omega_{c e}^{2}} \frac{\partial f_{e}}{\partial v_{e}} \mathrm{~d} v_{e}
\end{gathered}
$$

[11] Here, $\nu_{a v}$ represents the average modified collision frequency (the sum of $\nu_{\text {eff }}^{\mathrm{N}_{2}}$ and $\nu_{\text {eff }}^{\mathrm{O}_{2}}$ ), $\nu_{e}$ is the electron velocity, and $f_{e}$ is the electron velocity distribution function, which is assumed to remain Maxwellian [Stubbe and Kopka, 1977; Moore, 2007]. To account for HF absorption and refraction through the ionosphere, the plasma is divided into slabs $1-\mathrm{km}$ thick in altitude. The absorption rate of HF power is proportional to the imaginary component of the refractive index. The remaining power that exits one slab and enters the next is given by integrating the absorption through the slab and accounting for the $1 / r^{2}$ spreading where $r$ is the distance from the transmitter. The final result of the model is the modified Hall and Pedersen conductivity in each slab at altitudes from 60 to $90 \mathrm{~km}$.
[12] In order to reverse the nonlinear conversion between $\mathrm{HF}$ power and conductivity and minimize radiated harmonics, we begin with the assumption that the Hall conductivity is sinusoidally varying at some altitude, and determine the HF power modulation needed to obtain that waveform by running the forward model in reverse. We choose to make the Hall conductivity sinusoidal since previous studies showed that the Hall currents contribute more to the ELF source currents than the Pedersen currents [Barr and Stubbe, 1984; Cohen et al., 2008]. Since the ionospheric parameters change with altitude, the Hall conductivity can only be sinusoidal at a specified altitude. Equation (5) can be inverted numerically or by a lookup table to obtain $\nu_{e f f}$. From there, $T_{e}$ and $\frac{d T_{e}}{d t}$ can be calculated from equations (2) and (3) and $S$ can be determined from equation (1). $S$ is now the power waveform that must reach the plasma in order for the modified Hall conductivity to be sinusoidal. The amplitude of the specified sinusoid is determined by using the HF heating equations in the forward direction to determine the conductivity difference induced in the plasma at a particular altitude when heated by a full power beam. If, after inversion, the power density needed to achieve that sinusoidal conductivity variation is non-physical (greater than the power that can reach that altitude or negative), then the amplitude of the specified conductivity is iteratively reduced until the power density is valid. Finally, the absorption and spreading losses from propagation through the ionosphere are reversed by integrating the absorption downward from the target altitude and undoing the $1 / r^{2}$ spreading. The resulting power waveform is then used to modulate the HF power of the HAARP array.

[13] These waveforms will be referred to herein as inversesine (inv-sin) waveforms. A waveform designed to induce a sinusoidal variation in the Hall conductivity at $75 \mathrm{~km}$ for example, is referred to as a $75 \mathrm{~km}$ inv-sin waveform. Ideally, the waveform should minimize harmonic distortion at the altitude that dominates the radiated ELF signal. This altitude depends significantly on ionospheric density with higher densities corresponding to a lower altitude of peak modulation [Barr and Stubbe, 1984; Payne, 2007; Jin et al., 2011]. To increase the chances of reducing generated harmonics overall, we will generate multiple inv-sin waveforms, each optimized for a different altitude. Figure 1 shows an example of three of these inv-sin waveforms, optimized for 60,75 , and $90 \mathrm{~km}$. Also included for comparison are square waves with duty cycles of $20 \%$ and $40 \%$, as well as sinusoidal waveforms. In these experiments, sinusoidal modulation refers to a sinusoidal HF amplitude, in which case the HF power $(S$ in equation (1)) actually varies as sin-squared. Square-root of sine (sqrt-sin) modulation results in a sinusoidal HF power. We note that past studies have found that generated ELF amplitude scales with HF power, not HF amplitude. The invsin waveforms have narrower peaks, where the beam is on for a shorter time compared to sinusoidal modulation. This effectively compensates for the shorter cooling time constants in the ionosphere much the way square waves with duty cycles smaller than $50 \%$ were shown to be optimal in Cohen [2009].

\section{Numerical Modeling}

[14] To verify that the resulting HF power transmission waveforms do reduce or eliminate generated harmonic 
S, normalized

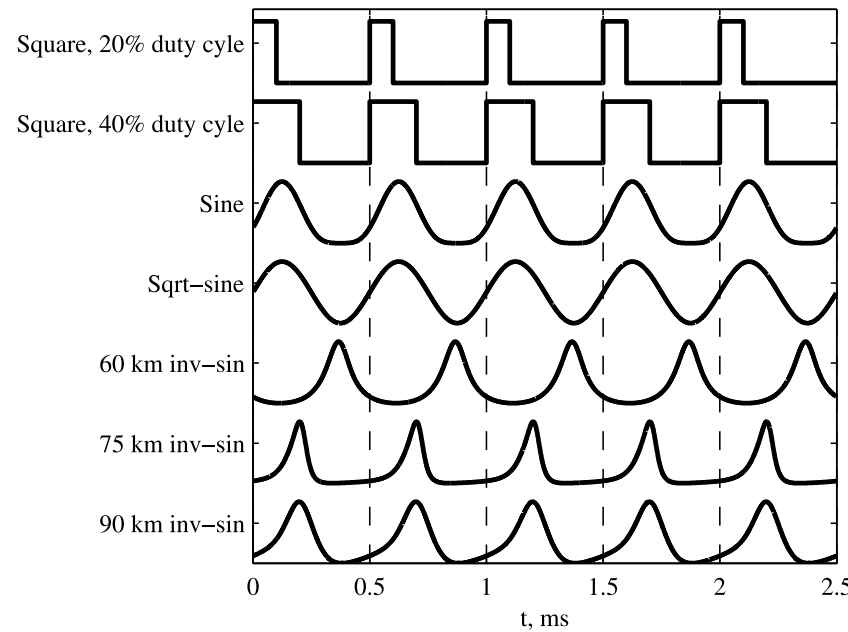

Figure 1. Examples of HF power transmission waveforms including square wave at two duty cycles, sine, sqrt-sine, and three inv-sin waveforms designed to minimize harmonics at 60, 75, and $90 \mathrm{~km}$.

content, they are run again through the forward model. The harmonic content of the resulting change in the Hall conductivity, $\Delta \sigma_{H}$ is examined and compared to sinusoidal modulation to verify that the inv-sin waveforms are suppressing harmonics as they were designed to.

[15] First we generate seven inv-sin waveforms optimized for altitudes from 60 to $90 \mathrm{~km}$ in $5 \mathrm{~km}$ increments using an electron density profile from the International Reference Ionosphere above $82 \mathrm{~km}$ and extrapolated to lower altitudes with an exponentially decaying density. This profile closely approximates an ionosphere given by Wait and Spies [1964] with parameters $h^{\prime}=84 \mathrm{~km}$ and $\beta=0.5 \mathrm{~km}^{-1}$ and is typical of a quiet nighttime ionosphere. The inv-sin waveforms are then simulated with the HF heating model in the forward direction under the same ionosphere and the results compared with sinusoidal modulation.

[16] Figure 2 shows the results of running an inv-sin waveform back through the forward model. Figure 2 a shows how the conductivity variation changes with altitude, where it becomes sinusoidal at $75 \mathrm{~km}$ as expected but is distorted at other altitudes. Panels $2 b$ and $2 c$ show the second and third harmonic of the modified Hall conductivity for three different modulation waveforms. The $75 \mathrm{~km}$ inv-sin waveform was meant to minimize harmonic distortion at an altitude of $75 \mathrm{~km}$ and does in fact show a null at that altitude. The second harmonic is $\sim 40 \mathrm{~dB}$ below the fundamental and the third harmonic is $\sim 56 \mathrm{~dB}$ below the fundamental. Sin and sqrt-sin modulation show more distortion with the second and third harmonic only $\sim 5-10 \mathrm{~dB}$ below the fundamental below 85 $\mathrm{km}$ with a minimum in distortion between $92-93 \mathrm{~km}$. Note that sqrt-sin modulation has higher harmonic content than sinusoidal modulation over almost all altitudes.

[17] However, inversion of the numerical HF heating model to create the inv-sin waveforms assumes knowledge of the ionospheric electron and neutral density profile as well as an ambient temperature profile. If the resulting waveforms are used when the actual ionosphere has a different profile from the one assumed, then the harmonic content of the ELF waves may be higher than expected. To examine the effect of an electron density profile that differs from the assumed one, we simulate waveforms generated using the assumed profile with electron density profiles different from the assumed profile. To create a family of electron density profiles for simulation, we use a simple two parameter model for ionospheric electron density [Wait and Spies, 1964]. This model
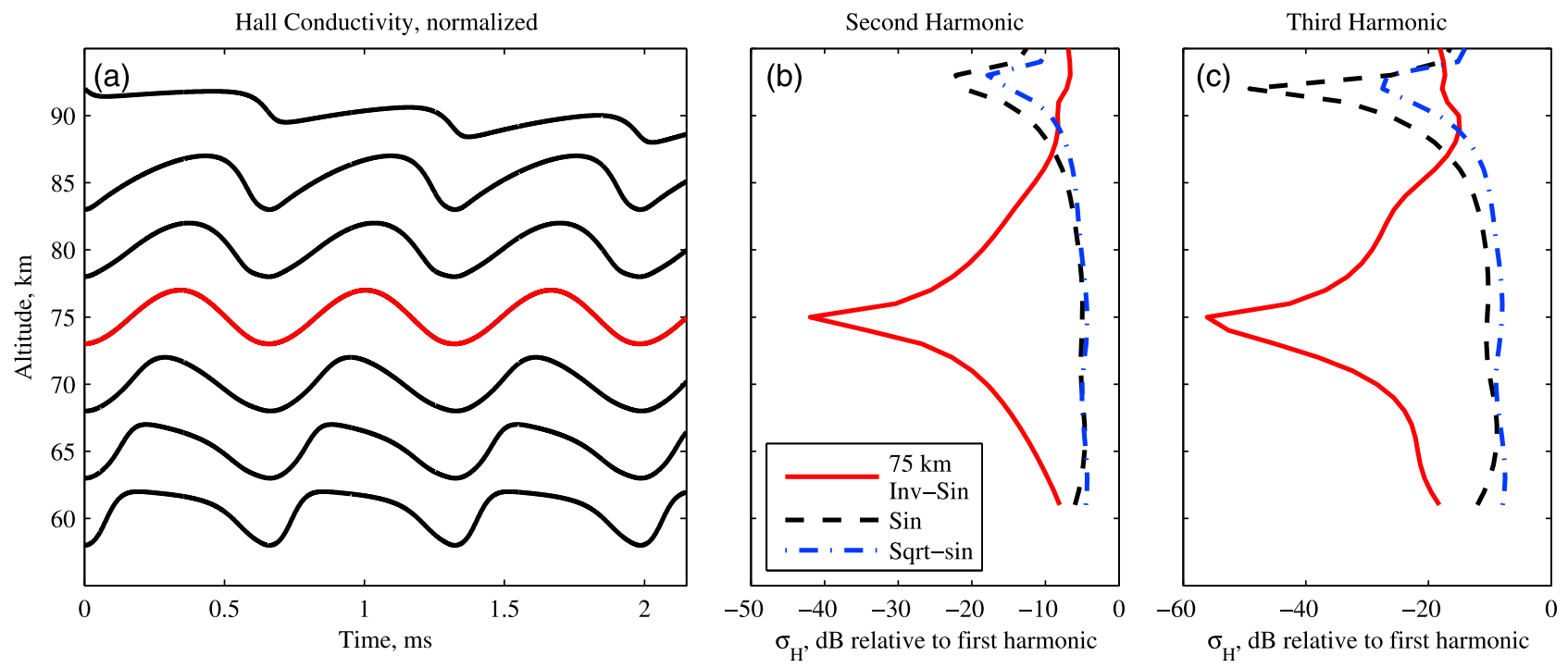

Figure 2. (a) Simulated Hall conductivity at several altitudes from 60 to $90 \mathrm{~km}$ when using a $75 \mathrm{~km}$ invsin waveform. Hall conductivity is sinusoidal at $75 \mathrm{~km}$ but not at other altitudes. (b) Second and (c) third harmonics of the modified Hall conductivity versus altitude in $\mathrm{dB}$ relative to the first harmonic. Harmonics at $75 \mathrm{~km}$ are suppressed substantially compared to sin and sqrt-sin modulation. Sqrt-sin has greater harmonic distortion compared to sin wave modulation. 


\begin{tabular}{c|c|c|c|c|c} 
& \multicolumn{6}{|c}{$\beta, \mathrm{km}^{-1}$} \\
$\mathrm{~h}, \mathrm{~km}$ & 0.3 & 0.4 & $\mathbf{0 . 5}$ & 0.6 & 0.7 \\
\hline 72 & -9.3 & -6.0 & -6.0 & -6.0 & -6.0 \\
78 & -11.4 & -16.1 & -15.9 & -6.0 & -6.0 \\
$\mathbf{8 4}$ & -29.4 & -35.9 & $-\mathbf{3 1 . 8}$ & -20.6 & -15.2 \\
90 & -18.5 & -18.1 & -17.9 & -17.8 & -17.7
\end{tabular}

Figure 3. Second harmonic of the Hall conductivity at $90 \mathrm{~km}$ expressed in $\mathrm{dB}$ relative to the first harmonic from simulations using a $90 \mathrm{~km}$ inv-sin waveform created assuming an ionospheric electron density expressed with $h^{\prime}=$ $84 \mathrm{~km}$ and $\beta=0.5 \mathrm{~km}^{-1}$.

represents an electron density that changes exponentially with altitude and is parameterized by $h^{\prime}$, an effective altitude, and $\beta$, a steepness parameter. The assumed electron density profile is one with $h^{\prime}=84 \mathrm{~km}$ and $\beta=0.5 \mathrm{~km}^{-1}$ and is used to generate inv-sin waveforms. These waveforms are then simulated on 20 electron density profiles with $h^{\prime}$ values of 72, 78, 84 , and $90 \mathrm{~km}$, and $\beta$ values of $0.3,0.4,0.5,0.6$, and $0.7 \mathrm{~km}^{-1}$. We examine the harmonic content produced when the inv-sin waveforms are used for each of the 20 different ionospheres to examine how closely $h^{\prime}$ and $\beta$ need to be to the assumed values in order for harmonic distortion to be suppressed relative to sinusoidal modulation.

[18] The results are given in Figure 3, which shows the ratio of the amplitude of the 2nd harmonic of the Hall conductivity at $90 \mathrm{~km}$ to the amplitude of the fundamental for a $90 \mathrm{~km}$ inv-sin waveform. This ratio is low for a matching ionosphere $\left(h^{\prime}=84 \mathrm{~km}, \beta=0.5 \mathrm{~km}^{-1}\right)$ as expected, but also remains below $-20 \mathrm{~dB}$ for other values of $\beta$ except $0.7 \mathrm{~km}^{-1}$. As long as $h^{\prime}$ is 84 or $90 \mathrm{~km}$, the second harmonic remains at least $-15 \mathrm{~dB}$ below the fundamental and there is less distortion at this altitude than with sinusoidal modulation (Figure 4). However, for smaller values of $h^{\prime}$, the second harmonic ratio increases to between $-16 \mathrm{~dB}$ to above $-10 \mathrm{~dB}$ for inv-sin modulation. Sinusoidal modulation generally has less distortion than $90 \mathrm{~km}$ inv-sin modulation when $h^{\prime}$ is below $84 \mathrm{~km}$, with the second harmonic roughly $24 \mathrm{~dB}$ below the fundamental for $h^{\prime}=78 \mathrm{~km}$ and $\beta$ between 0.3 and $0.5 \mathrm{~km}^{-1}$. The harmonic content is not particularly sensitive to $\beta$ for either modulation waveform as values between 0.3 and $0.5 \mathrm{~km}^{-1}$ do not affect the harmonic content appreciably.

[19] Figure 5 shows an example of the simulated Hall conductivity when an $90 \mathrm{~km}$ inv-sin waveform designed for a specific value of $h^{\prime}$ is applied to an ionosphere for which the actual value of $h^{\prime}$ is smaller. The plots on the left side show the change in Hall conductivity at $90 \mathrm{~km}$ and $85 \mathrm{~km}$ for the expected $h^{\prime}=84 \mathrm{~km}, \beta=0.5 \mathrm{~km}^{-1}$ ionosphere. At $90 \mathrm{~km}$ the conductivity appears sinusoidal and distortion free. However, the plots on the right side, which show the conductivity when $h^{\prime}=78 \mathrm{~km}, \beta=0.4 \mathrm{~km}^{-1}$, are notably distorted. Because the ionosphere on the right hand side is more dense at lower altitudes, much of the absorption happens near $85 \mathrm{~km}$ and the amplitude of the conductivity at that altitude is greater than for the less dense ionosphere. This leaves less power available at $90 \mathrm{~km}$, which has a much smaller change in conductivity. The electron density itself does not affect the heating and cooling time constants [Cohen, 2009, p. 46], but the assumptions inherent in calculating the inv-sin waveform assume that a certain power density will be absorbed before reaching $90 \mathrm{~km}$, which if not correct, results in a distorted conductivity change at that altitude.

\section{Experimental Results}

\subsection{Harmonic Minimization}

[20] The inv-sin waveforms were transmitted as part of an experiment conducted at HAARP from July 22-26, 2011 for a total of 3 hours of transmission time. The 1-minute format consists of $20 \mathrm{~s}$ at ELF frequencies of 510, 1510, and $2125 \mathrm{~Hz}$. For each frequency, 10 amplitude modulation waveforms were transmitted for $2 \mathrm{~s}$ each. These were: square wave with $40 \%$ duty cycle, sinusoidal $\mathrm{HF}$ amplitude (power varies as sine squared), sqrt-sine HF amplitude (sinusoidal HF power), and seven inverted sine formats optimized for altitudes from 60 $90 \mathrm{~km}$ in $5 \mathrm{~km}$ increments. The assumed ionospheric density profile was taken from the International Reference Ionosphere above $82 \mathrm{~km}$ and extrapolated to lower altitudes assuming an exponential decay. This is the same standard nighttime profile from section 3. A full power $2.75 \mathrm{MHz}, \mathrm{X}$-mode $\mathrm{HF}$ beam was used for the entire experiment.

[21] ELF measurements were made with the Stanford AWESOME receiver [Cohen et al., 2010c] located at Chistochina, Alaska, $37 \mathrm{~km}$ northeast of HAARP. Signals from two orthogonal loop antennas are sampled at $100 \mathrm{kHz}$ and digitized with 16 bits of resolution and $<200 \mathrm{~ns}$ timing error. ELF amplitudes at the carrier and harmonic frequencies are extracted from a Fast Fourier Transform of the data and amplitudes from the two antennas are combined to obtain the total horizontal component of the wave magnetic field.

[22] Signal intensities were strongest during transmissions on July 23 from 0906-0936 UT. The results in Figure 6 show the amplitude of each harmonic up to the 10th, relative to the fundamental, for 60 and $90 \mathrm{~km}$ inv-sin, as well as sinusoidal modulation. The 60 and $90 \mathrm{~km}$ inv-sin waveforms performed the best of the inv-sin waveforms but still did not result in lower harmonic content of the received ELF waves when compared to sinusoidal modulation. The second harmonic was over $20 \mathrm{~dB}$ below the fundamental for sinusoidal modulation but only $10-13 \mathrm{~dB}$ below for the inv-sin waveforms. In the third harmonic, $90 \mathrm{~km}$ inv-sin does show a substantial improvement over sine wave $(-29 \mathrm{~dB}$ versus $-23 \mathrm{~dB}$ ). In the higher harmonics, $60 \mathrm{~km}$ inv-sin and sine perform similarly with $90 \mathrm{~km}$ inv-sin $\sim 3 \mathrm{~dB}$ worse. These results suggest that the ionospheric density profile on the day of the experiment did not match the profile assumed when generating the inv-sin waveform.

\begin{tabular}{c|c|c|c|c|c} 
& \multicolumn{5}{|c}{$\beta, \mathrm{km}^{-1}$} \\
$\mathrm{~h}, \mathrm{~km}$ & 0.3 & 0.4 & 0.5 & 0.6 & 0.7 \\
\hline 72 & -2.8 & -6.0 & -6.0 & -6.0 & -6.0 \\
78 & -24.5 & -25.2 & -23.9 & -6.0 & -6.0 \\
84 & -9.1 & -9.4 & -10.0 & -11.2 & -14.2 \\
90 & -7.9 & -7.8 & -7.7 & -7.7 & -7.7
\end{tabular}

Figure 4. Second harmonic of the Hall conductivity at $90 \mathrm{~km}$ expressed in $\mathrm{dB}$ relative to the first harmonic from simulations using a sinusoidal waveform. Distortion is generally lower than $90 \mathrm{~km}$ inv-sin for $h^{\prime}$ below $84 \mathrm{~km}$ and greater for $h^{\prime}$ of $84 \mathrm{~km}$ and above. 

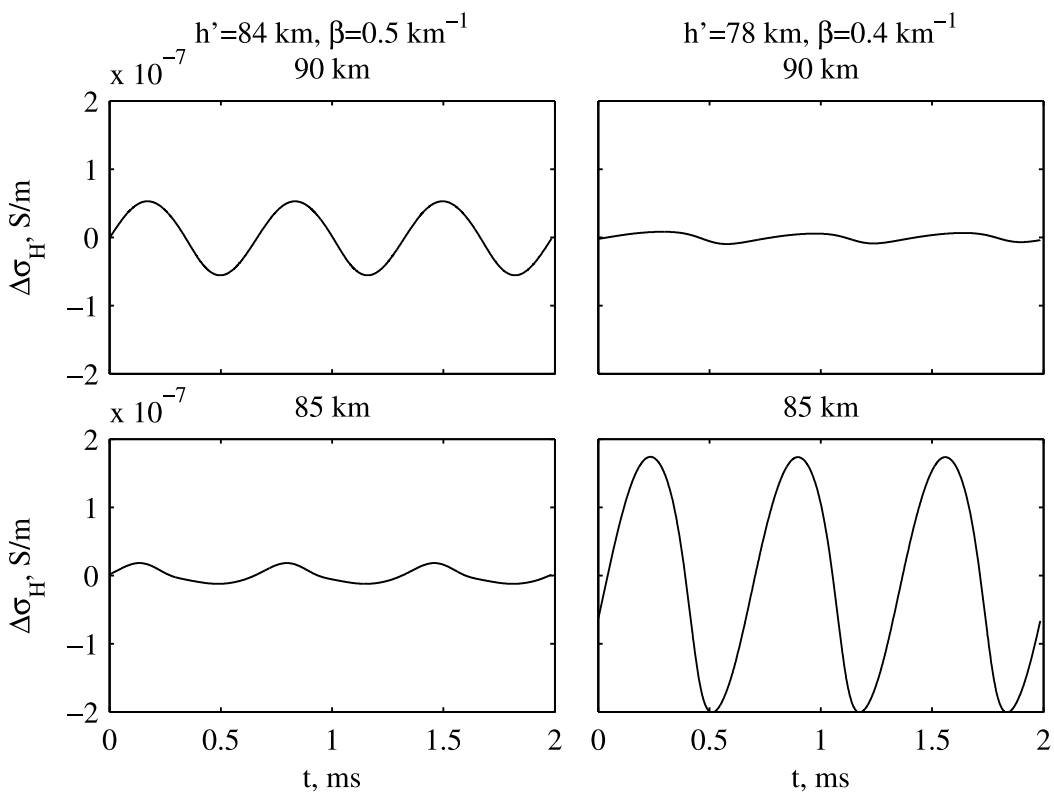

Figure 5. Simulated change in Hall conductivity when using a $90 \mathrm{~km}$ inv-sin waveform assuming an ionosphere modeled by $h^{\prime}=84 \mathrm{~km}$ and $\beta=0.5 \mathrm{~km}^{-1}$. Plots on the left show the conductivity at 90 and $85 \mathrm{~km}$ with a matching ionosphere where the conductivity at $90 \mathrm{~km}$ is distortion free. Plots on the right show the conductivity at 90 and $85 \mathrm{~km}$ with a mismatched ionosphere. Most of the HF power has been absorbed at lower altitudes and the modulation at $90 \mathrm{~km}$ is much weaker and more distorted.

\subsection{Total Power and Efficiency}

[23] In addition to examining the relative amplitudes of harmonics, we also compare the power in the fundamental as well as the total power including harmonics for different modulation waveforms. Since different waveforms require different amounts of power to transmit we also compare the efficiency of various waveforms and define efficiency as the ratio of the received power to the power in the waveform. The power in the HF amplitude waveform $x(t)$ is defined as:

$$
\frac{1}{T} \int_{0}^{T} x^{2}(t) \mathrm{d} t
$$

where $T$ is the ELF fundamental period. Experimental data for inv-sin waveforms were taken from the same experiment as above. In addition a separate experiment using squarewave modulation with duty cycles from 15 to $85 \%$ in $5 \%$ increments was also performed from October 2, 5, 9, and 13, 2010 with the best data from $0830-0900$ UT on October 13 . For this experiment data were taken from an AWESOME receiver near Paxson, Alaska roughly $50 \mathrm{~km}$ north of HAARP.

[24] In the duty-cycle experiment, 1 second tones at frequencies of 510, 990, 1530, 2010,3030, and $5010 \mathrm{~Hz}$ are transmitted at each of 15 different duty cycles. This sequence is repeated using $100 \%, 50 \%, 25 \%$, and $12.5 \%$ power. The entire format thus repeats every $6 \mathrm{~min}$. We will consider only a full-power beam modulated at $2010 \mathrm{~Hz}$ and compare the received signal to the results from the inv-sin experiment at $2125 \mathrm{~Hz}$. Both the inv-sin and duty cycle experiments contain a tone using square-wave modulation with a $40 \%$ duty cycle. Thus to compare modulation waveforms across both experiments and to normalize for different natural conditions, received power will always be reported as relative to the received power when $40 \%$ duty cycle square wave modulation is used. For example, sine wave modulation has a total received power of 0.55 , meaning the average measured power across all harmonics is $55 \%$ of the average measured power across all harmonics when $40 \%$ duty cycle square wave modulation was used. Similarly, a fundamental power of 0.81 for sine wave modulation means that the average received power in the fundamental is $81 \%$

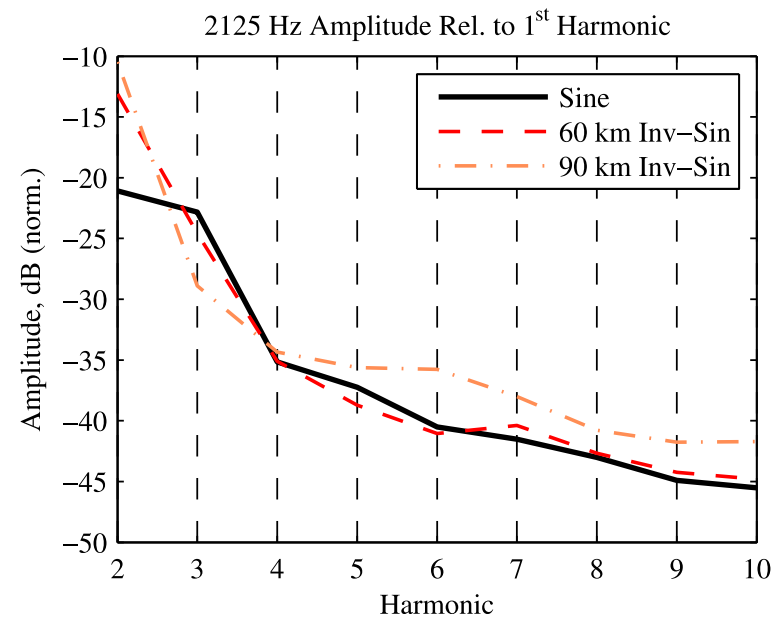

Figure 6. Harmonic content of ELF signals received in Chistochina, Alaska for $2125 \mathrm{~Hz}$ signals generated using Sine wave modulation (black), and $60 \mathrm{~km}$ and $90 \mathrm{~km}$ invsin modulation (red and orange). Amplitudes are in $\mathrm{dB}$ relative to the fundamental. The inv-sin waveforms do not have substantially lower harmonic content compared to sine wave. 

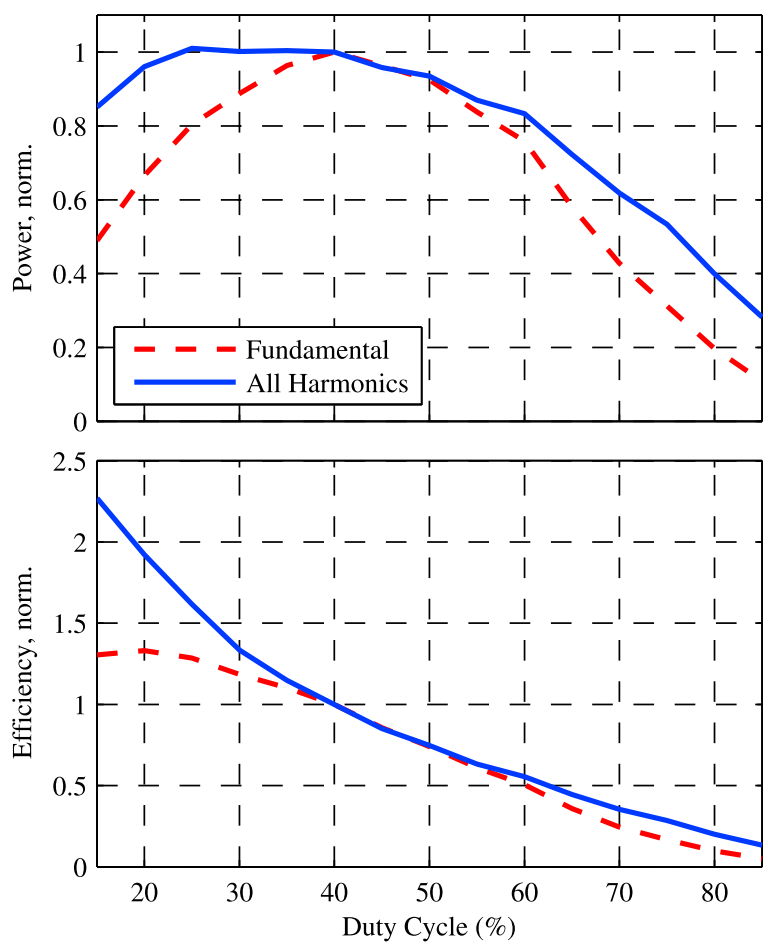

Figure 7. Received power and efficiency for $2010 \mathrm{~Hz}$ signals generated using square wave modulation of different duty cycles and received at Paxson, Alaska. Red line only includes received power in the fundamental; blue line includes received power in the first five harmonics. All values are normalized so that $40 \%$ duty cycle has a value of 1 . A $40 \%$ duty cycle yielded the most power in the fundamental, while duty cycles between $25-40 \%$ have the most total power. Total power efficiency increases for lower duty cycles and fundamental efficiency peaks at $20 \%$ duty cycle.

of the average received power in the fundamental with square wave modulation. Efficiency is also normalized in the same way. Figure 7 shows how the fundamental and total received power and efficiency vary as a function of duty cycle. Peak power in the fundamental occurs with a duty cycle of $40 \%$ and drops rapidly for other duty cycles. When considering all harmonics, there is a broader maximum in received power with duty cycles from $25-40 \%$. Thus total efficiency is highest for the smallest duty cycle used (15\%) since the received power only decreases slightly (about $85 \%$ of the highest total power) while the power needed to transmit the waveform decreases. However, when considering only the fundamental, the efficiency peaks at $20 \%$ duty cycle. These results are very similar to the ones in Cohen [2009], which also showed a peak in the fundamental power near a $40 \%$ duty cycle but a peak in fundamental efficiency at $15 \%$ duty cycle in measurements at Chistochina, Alaska.

[25] The results of the duty-cycle and inv-sin experiments are summarized in Table 1. Square wave modulation with duty cycles below $40 \%$ generally produce the strongest ELF waves and have high efficiency. Received signals are strongest for a duty cycle of $40 \%$, regardless of whether only the fundamental or all harmonics are considered. A duty cycle of $20 \%$ resulted in the highest efficiency in the sense of highest received fundamental power relative to the total input power. Although the lower duty cycle generates only 0.67 times the power in the fundamental as for a $40 \%$ duty cycle, it only requires half the power to transmit, resulting in a 1.33 times increase in efficiency.

[26] Variants of sinusoidal modulation all had lower levels of harmonic distortion (indicated by a greater percentage of the total power contained in the fundamental), than square wave modulation. Of these variations, sinusoidal modulation had the smallest level of harmonic distortion, with $98.7 \%$ of the power in the fundamental. The $60 \mathrm{~km}$ inv-sin waveform had slightly higher distortion with $94.8 \%$ of the power in the fundamental, but is also more efficient $(0.81$ versus 0.70 for the fundamental power). Sqrt-sine, which results in a sinusoidally varying HF power, performs worse in all respects compared to sinusoidal modulation.

\section{Discussion}

[27] Generally, the time constants for heating of the electrons by absorption of HF wave energy are faster than for cooling by collisions with neutral molecules. This discrepancy can explain several of the features we observed in our experiments with different modulation waveforms. First, any attempt to create an HF power waveform that produces a sinusoidal conductivity variation must compensate for the asymmetry in the heating and cooling time constants. Thus it makes sense that the inv-sin waveforms in Figure 1 have a slower risetime and faster fall time, which helps to decrease the heating and increase the cooling time constant so that they are balanced. This is most apparent for the $75 \mathrm{~km}$ invsin waveform where the fall time is especially fast compared

Table 1. Experimental Data for 5 Different Waveforms Including Power in Waveform, Received Fundamental Power and Efficiency, Received Total Power and Efficiency, and Fraction of Received Power in the Fundamental ${ }^{\mathrm{a}}$

\begin{tabular}{|c|c|c|c|c|c|c|}
\hline \multirow[b]{2}{*}{ Waveform } & \multirow[b]{2}{*}{ Input Power } & \multicolumn{2}{|c|}{ Fundamental } & \multicolumn{2}{|c|}{ All Harmonics } & \multirow[b]{2}{*}{ Fraction in Fundamenta } \\
\hline & & Power & Efficiency & Power & Efficiency & \\
\hline $40 \%$ Square & 0.40 & 1 & 1 & 1 & 1 & 0.85 \\
\hline $20 \%$ Square & 0.20 & 0.67 & 1.33 & 0.96 & 1.92 & 0.59 \\
\hline Sine & 0.375 & 0.65 & 0.70 & 0.55 & 0.58 & 0.987 \\
\hline $60 \mathrm{~km}$ Inv-Sin & 0.27 & 0.55 & 0.81 & 0.48 & 0.71 & 0.948 \\
\hline Sqrt-Sin & 0.50 & 0.40 & 0.32 & 0.37 & 0.30 & 0.902 \\
\hline
\end{tabular}

${ }^{\mathrm{a}}$ Received power and efficiency are referenced against $40 \%$ Square Wave. $40 \%$ square wave has the highest received power, $20 \%$ square wave has the highest efficiency, and sine wave has the lowest harmonic distortion. $60 \mathrm{~km}$ inv-sin has slightly higher distortion than sine wave but is more efficient. SqrtSin is worse than sine in every metric. 
to the risetime. Note that the $60 \mathrm{~km}$ and $90 \mathrm{~km}$ inv-sin waveforms more closely approximate a sine wave. At lower altitudes, the neutral density is much higher so the electrons will cool more rapidly and the heating and cooling time constants are better balanced. As the altitude increases, the cooling rate decreases. However, at very high altitudes, most of the HF power has been absorbed and both the heating and cooling rates have decreased substantially and are again more balanced. This may explain why the 60 and $90 \mathrm{~km}$ invsin waveforms generated less distortion in experiments than the intermediate altitude inv-sin waveforms as 60 and $90 \mathrm{~km}$ inv-sin are the most symmetric and most closely resemble a sinusoid. Because sinusoidal modulation already creates little harmonic distortion near $92 \mathrm{~km}$ (Figures $2 \mathrm{~b}$ and 2c), it may be that it is already near optimal for minimizing harmonics at that altitude.

[28] The smaller harmonic distortion of sinusoidal modulation versus inv-sin modulation in experiments is likely the result of an ionospheric density profile that did not match the assumed profile as the simulations in Section 3 showed. The discrepancy may also be a result of an incomplete HF heating model that does not accurately reproduce the harmonic content observed in experimental data even if the ionospheric density profile used in the model is similar to the actual ionosphere. For example, the nonlinearity and filtering that occurs within the HAARP transmitting hardware and the reflection of the HF waves between different layers in the ionosphere are not included in the current model. Although these effects are likely small compared to ionospheric variability, they could be incorporated into future models to make them more complete.

[29] However, all the inv-sin waveforms also take less power to transmit because the power is high for a shorter fraction of an ELF period. For example $60 \mathrm{~km}$ inv-sin has an effective duty cycle of $27 \%$ compared to $37.5 \%$ for sine wave. The heating only needs to occur for a short period for the electron temperature to increase to saturation since the heating time constant is fast. Once the electron temperature is near saturation, the HF power can be reduced rapidly, allowing more time for the temperature to recover to its ambient value with the slower cooling time constant. This effect explains why square waves with smaller duty cycles generate stronger ELF waves than ones with higher duty cycles. A $40 \%$ duty cycle square wave generated the strongest ELF waves in the fundamental, and smaller duty cycles result in only a small decrease in received power. Duty cycles larger than $40 \%$ generate less power and are much less efficient because they result in longer heating and shorter cooling periods, which heat the electrons to saturation but do not allow them to cool very much before the next heating period begins.

[30] This preference for smaller duty cycles also explains why sqrt-sin modulation is worse than sinusoidal modulation in all respects. Sinusoidal modulation means the HF power is proportional to sin squared, which has a lower effective duty cycle than sin (more time at low power than at high power). It may be interesting in future experiments to try waveforms represented by an HF amplitude of $\sin ^{k}(2 \pi f t)$ where $k$ is some number greater than 0.5 . Higher values of $k$ produce smaller effective duty cycles, which may improve efficiency while still maintaining low values of harmonic distortion compared to square wave. These waveforms would also be easier to implement than the inv-sin waveforms.

\section{Conclusion}

[31] Generation of ELF waves using amplitude modulation of an HF beam results in harmonic distortion due to the nonlinear relationship between HF power and conductivity change in the ionosphere. With a sinusoidal amplitude envelope, $98.7 \%$ of the received ELF power is in the fundamental. A sinusoidal power envelope has worse distortion with only $90.2 \%$ of the power in the fundamental. To minimize the harmonic distortion we used numerical models to compute a non-sinusoidal HF envelope that would result in a sinusoidal Hall conductivity at a given altitude. In simulation these waveforms do minimize harmonic distortion at a specific altitude but they are sensitive to ionospheric electron density profiles. The inv-sin waveforms generally perform as intended when the actual ionosphere has a similar or greater value of $h^{\prime}$ as the one assumed when generating the waveforms. Otherwise, the inv-sin waveform may result in greater harmonic distortion than sinusoidal modulation. This was the result in experiments where the $60 \mathrm{~km}$ inv-sin waveform produced the least distortion of all the inv-sin waveforms but still resulted in only $94.8 \%$ of the received power in the fundamental.

[32] However the choice of waveform also affects the received power of the ELF wave. Square wave modulation, though it results in more harmonic distortion $(85 \%$ of power in the fundamental for a $40 \%$ duty cycle) is often used because it generates the most absolute power in the fundamental. Smaller duty cycles trade off a small decrease in received power for a larger savings in the average power needed to transmit the waveform. A duty cycle of $20 \%$ was most efficient in the sense of maximizing the received power in the fundamental versus the power needed to transmit. If considering the power in all the harmonics, even smaller duty cycles are more efficient. Although the inv-sin waveforms resulted in more harmonic distortion than sinusoidal modulation, they require less power to transmit and are more efficient while still having reasonably low levels of distortion. In general, there appears to be a tradeoff between harmonic distortion and efficiency when choosing waveforms.

[33] Acknowledgments. This research has been carried out with support from HAARP, Office of Naval Research, and Air Force Research Laboratory, via ONR award N0014-09-1-0100 and AFRL award FA945311-C-0011 to Stanford University, and by a Stanford Graduate Fellowship. We also thank Mike McCarrick and Helio Zwi for operating the HAARP array and Ed Kennedy for organizing the SSRC experimental campaign that led to these observations.

[34] Robert Lysak thanks the reviewers for their assistance in evaluating this paper.

\section{References}

Banks, P. (1966), Collision frequencies and energy transfer electrons, Planet. Space Sci., 14, 1085-1103, doi:10.1016/0032-0633(66)90024-9.

Barr, R., and P. Stubbe (1984), ELF and VLF radiation from the "polar electrojet antenna," Radio Sci., 19, 1111-1122, doi:10.1029/RS019i004p01111.

Barr, R., and P. Stubbe (1993), ELF harmonic radiation from the Troms $\varnothing$ heating facility, Geophys. Res. Lett., 20, 2243-2246, doi:10.1029/ 93GL02495.

Bernstein, S. L., M. L. Burrows, J. E. Evans, A. S. Griffiths, D. A. McNeill, C. W. Niessen, I. Richer, D. P. White, and D. K. Willim (1974), Longrange communications at extremely low frequencies, Proc. IEEE, 62(3), 292-312, doi:10.1109/PROC.1974.9426. 
Cohen, M. B. (2009), ELF/VLF phased array generation via frequencymatched steering of a continuous HF ionospheric heating beam, PhD thesis, Stanford Univ., Stanford, Calif.

Cohen, M. B., M. Gołkowski, and U. S. Inan (2008), Orientation of the HAARP ELF ionospheric dipole and the auroral electrojet, Geophys. Res. Lett., 35, L02806, doi:10.1029/2007GL032424.

Cohen, M. B., U. S. Inan, M. Gołkowski, and N. G. Lehtinen (2010a), On the generation of ELF/VLF waves for long-distance propagation via steerable HF heating of the lower ionosphere, J. Geophys. Res., 115, A07322, doi:10.1029/2009JA015170.

Cohen, M. B., U. S. Inan, M. Gołkowski, and M. J. McCarrick (2010b), ELF/VLF wave generation via ionospheric HF heating: Experimental comparison of amplitude modulation, beam painting, and geometric modulation, J. Geophys. Res., 115, A02302, doi:10.1029/2009JA014410.

Cohen, M. B., U. S. Inan, and E. W. Paschal (2010c), Sensitive broadband ELF/VLF radio reception with the AWESOME instrument, IEEE Trans. Geosci. Remote Sens., 48, 3-17, doi:10.1109/TGRS.2009.2028334.

Dalgarno, A., M. B. McElroy, M. H. Rees, and J. C. G. Walker (1968), The effect of oxygen cooling on ionospheric electron temperatures, Planet. Space Sci., 16, 1371-1380, doi:10.1016/0032-0633(68)90141-4.

Getmantsev, G. G., N. A. Zuikov, D. S. Kotik, L. F. Mironenko, N. A. Mitiakov, V. O. Rapoport, I. A. Sazonov, V. I. Trakhtengerts, and V. I. Eidman (1974), Combination frequencies in the interaction between high-power short-wave radiation and ionospheric plasma, Sov. JETP Lett., Engl. Transl., 20, 229-232.

Gołkowski, M., U. S. Inan, A. R. Gibby, and M. B. Cohen (2008), Magnetospheric amplification and emission triggering by ELF/VLF waves injected by the 3.6 MW HAARP ionospheric heater, J. Geophys. Res., 113, A10201, doi:10.1029/2008JA013157.

Hedin, A. E. (1991), Extension of the MSIS thermosphere model into the middle and lower atmosphere, J. Geophys. Res., 96, 1159-1172, doi:10.1029/90JA02125.

James, H. G. (1985), The ELF spectrum of artificially modulated D/Eregion conductivity, J. Atmos. Terr. Phys., 47, 1129-1142.

James, H. G., R. L. Dowden, M. T. Rietveld, P. Stubbe, and H. Kopka (1984), Simultaneous observations of ELF waves from an artificially modulated auroral electrojet in space and on the ground, J. Geophys. Res., 89, 1655-1666, doi:10.1029/JA089iA03p01655.

Jin, G., M. Spasojevic, M. B. Cohen, U. S. Inan, and N. G. Lehtinen (2011), The relationship between geophysical conditions and ELF amplitude in modulated heating experiments at HAARP: Modeling and experimental results, J. Geophys. Res., 116, A07310, doi:10.1029/2011JA016664.

Kapustin, I. N., R. A. Pertsovskiľ, A. N. Vasil'Ev, V. S. Smirnov, O. M. Raspopov, L. E. Solov'eva, A. A. Ul'Yachenko, A. A. Arykov, and N V. Galakhova (1977), Generation of radiation at combination frequencies in the region of the auroral electric jet, Sov. JETP Lett., Engl. Transl., 25, $228-231$.
Mentzoni, M. H., and R. V. Row (1963), Rotational excitation and electron relaxation in nitrogen, Phys. Rev., 130, 2312-2316, doi:10.1103/ PhysRev.130.2312.

Milikh, G. M., K. Papadopoulos, M. McCarrick, and J. Preston (1999), ELF emission generated by the HAARP HF-heater using varying frequency and polarization, Radiophys. Quantum Electron., 42, 639-646, doi:10.1007/BF02676849.

Moore, R. C. (2007), ELF/VLF wave generation by modulated HF heating of the auroral electrojet, PhD thesis, Stanford Univ., Stanford, Calif.

Moore, R. C., U. S. Inan, and T. F. Bell (2006), Observations of amplitude saturation in ELF/VLF wave generation by modulated HF heating of the auroral electrojet, Geophys. Res. Lett., 33, L12106, doi:10.1029/ 2006GL025934.

Oikarinen, A., J. Manninen, J. Kultima, and T. Turunen (1997), Observations of intensity variations and harmonics of heater induced VLF waves, J. Atmos. Sol. Terr. Phys., 59, 2351-2360.

Papadopoulos, K., T. Wallace, M. McCarrick, G. M. Milikh, and X. Yang (2003), On the efficiency of ELF/VLF generation using HF heating of the auroral electrojet, Plasma Phys. Rep., 29, 561-565.

Papadopoulos, K., C.-L. Chang, J. Labenski, and T. Wallace (2011), First demonstration of HF-driven ionospheric currents, Geophys. Res. Lett., 38, L20107, doi:10.1029/2011GL049263.

Payne, J. A. (2007), Spatial structure of very low frequency modulated ionospheric currents, Ph.D. thesis, Stanford Univ., Stanford, Calif.

Rietveld, M. T., H.-P. Mauelshagen, P. Stubbe, H. Kopka, and E. Nielsen (1987), The characteristics of ionospheric heating-produced ELF/VLF waves over 32 hours, J. Geophys. Res., 92, 8707-8722.

Rodriguez, J. V. (1994), Modification of the Earth's ionosphere by verylow-frequency transmitters, PhD thesis, Stanford Univ., Stanford, Calif.

Sen, H. K., and A. A. Wyller (1960), On the generalization of the AppletonHartree magnetoionic formulas, J. Geophys. Res., 65, 3931-3950, doi:10.1029/JZ065i012p03931.

Stubbe, P., and H. Kopka (1977), Modulation of polar electrojet by powerful HF waves, J. Geophys. Res., 82, 2319-2325, doi:10.1029/ JA082i016p02319.

Stubbe, P., and W. S. Varnum (1972), Electron energy transfer rates in the ionosphere, Planet. Space Sci., 20, 1121-1126, doi:10.1016/0032-0633 (72)90001-3.

Stubbe, P., et al. (1982), Ionospheric modification experiments in northern Scandinavia, J. Atmos. Terr. Phys., 44, 1025-1029.

Tomko, A. A., A. J. Ferraro, and H. S. Lee (1980), D region absorption effects during high-power radio wave heating, Radio Sci., 15, 675-682.

Wait, J. R., and K. Spies (1964), Characteristics of the Earth-ionosphere waveguide for VLF radio waves, Tech. Note 300, Natl. Bur. of Stand., Gaithersburg, Md. 